\title{
DIFERENCIAS TIPOLÓGICAS Y ADQUISICIÓN DE SEGUNDAS LENGUAS II: LAS CONSTRUCCIONES ATRIBUTIVAS, LOCATIVAS, EXISTENCIALES Y POSESIVAS*
}

\author{
Lluïsa Gràcia, Berta Grous y Laura Garganta \\ Universitat de Girona
}

\section{RESUMEN}

El objetivo principal de este artículo es analizar cómo influyen las diferencias tipológicas en la adquisición de las construcciones atributivas, locativas, existenciales y posesivas, y cuál es el papel de las estrategias generales de simplificación que se han propuesto para explicar algunas omisiones de las formas copulativas. Los datos analizados provienen de un estudio transversal en el que han sido entrevistados aprendices de catalán y de español cuyas primeras lenguas son el árabe marroquí, el chino o el rumano.

A partir de los datos recogidos, se ha observado que los aprendices con un nivel más bajo de cada grupo producen un número más elevado de omisiones que los de un nivel más avanzado, independientemente de si en la primera lengua existe o no una forma copulativa en estas construcciones. A pesar de ello, las diferencias entre las características de la producción de cada grupo en estadios más avanzados solo pueden ser explicadas a partir de las propiedades de la primera lengua, tanto las relacionadas con las construcciones analizadas como aquellas relativas a la flexión verbal. También se han detectado otros factores que pueden influir en este proceso, principalmente algunas características especiales de las segundas lenguas, las diferencias entre el catalán y el español en algunas construcciones (todos los aprendices están expuestos a ambas lenguas), y las relaciones y los límites no siempre claros entre los cuatro tipos de estructuras en todas las lenguas.

Palabras clave: tipología, adquisición de segundas lenguas, atributivas, locativas, existenciales, posesivas, paradigma locativo, cópula, flexión verbal, catalán, español, chino, árabe, rumano.

\footnotetext{
* Este trabajo se ha beneficiado de tres ayudas del Ministerio de Ciencia y Tecnología (BFF2003-04043, HUM2006-07217/FILO y AP2006-0260) y una de la Generalitat de Catalunya (2006FIC 00840). Agradecemos la colaboración de todas las personas que durante varios meses han echo posible este trabajo, especialmente a los investigadores y colaboradores del Gabinet d'Assessorament Lingüistic per a la Immigració de la Universitat de Girona y a M. Mas y a los profesores del Consorci per a la Normalització Lingüística que nos han facilitado el contacto con las personas entrevistadas, a quienes también agradecemos su colaboración. A efectos de la evaluación de la investigación por los órganos competentes, L. Gràcia ha realizado el análisis de los datos y la redacción del artículo y han coordinado las tareas de transcripción y codificación de las entrevistas. B. Crous y L. Garganta han participado en el proceso de entrevistar a los informantes, han asumido los trabajos de transcripción y de codificación y han colaborado en el análisis y la discusión de los resultados.
} 


\section{Abstract}

The main aim of this article is to analyse the way in which the acquisition of attributive, locative, existential and possessive constructions is influenced by typological differences, and which role plays in this process the general strategies of simplification proposed to explain some omissions of the copulative forms. The data analysed come from a transversal study in which students of Catalan and Spanish, whose first languages are Moroccan Arabic, Chinese or Romanian, have been interviewed.

It has been observed from the data collected that learners from a lower level in any of the three groups produce a higher number of verb omissions than the ones from a more advanced level, independently of whether or not there is a copulative form in those particular constructions in their first language. Nonetheless, the differences between the characteristics of the production of each group in more advanced stages can only be explained through the properties of the first language, both those related to the constructions analysed and those connected to the type of verbal inflection. Other factors that can have an influence on that process have also been detected, mainly some special characteristics of the second languages, the differences between Catalan and Spanish in some constructions (all learners are exposed to both languages), and the relationships and the sometimes unclear limits between the four types of structures in all languages.

Key Words: Typology, L2 Learning, Attributive Sentences, Locative Sentences, Posesive Sentences, Existencial Sentences, Locative Paradigm, Copula, Verbal inflection, Catalan, Spanish, Chinese, Arabic, Romanian.

\section{INTRODUCCIÓN}

Es un lugar común en los estudios de lingüística general que las oraciones atributivas, locativas, existenciales y posesivas (ALEP) no se construyen igual en todas las lenguas. El tema ha sido tratado por muchos autores, desde los trabajos clásicos de Benveniste 1950 y 1960, de Allen 1964 o de Lyons 1967, hasta la gramática generativa, que, desde los primeros tiempos, ha intentado dar cuenta de estas relaciones formulando estructuras profundas idénticas o muy similares (cf. Bach 1967, Fillmore 1966, Clark 1978, Jackendoff 1974 y 1976, etc.). Para Freeze 1992, por ejemplo, las locativas, las existenciales y las posesivas derivan de una misma estructura inicial y las cópulas no se consideran elementos léxicos sino la realización de un rasgo locativo de Flexión.

Aunque en español y en catalán, excepto en casos especiales, estas oraciones llevan siempre un elemento verbal explícito (ser, estar, haber/ haver-hi, tener/tenir), esta no es la situación general en las lenguas del mundo. Así, es muy frecuente encontrar lenguas en las que estas construcciones no contienen ningún verbo explícito: nunca (por ejemplo, dialonké), o en determinadas circunstancias o construcciones (ruso, árabe, chino). También es usual que el número de elementos léxicos de tipo verbal con los que se forman sea mucho más reducido que el del catalán o del español, que, junto con el portugués, son un caso especial incluso dentro de las lenguas románicas: tienen una única cópula el 
gaélico irlandés, el japonés, el hindi y el penyabí, y dos, el francés, el italiano, el rumano y el inglés².

Dado que estos cuatro tipos de construcciones están relacionados semánticamente, a menudo se agrupan las locativas (Los libros están aquí), existenciales (Aqui hay libros) y posesivas (Nosotros tenemos libros) en el llamado «paradigma locativo» (cf. Freeze 1992): en todos los casos, se trata de predicados que expresan un estado en el que siempre participa una entidad cuya locación se predica explícita o implícitamente. En las posesivas el poseedor puede concebirse como el lugar donde se localiza la entidad. En el caso de las atributivas, esta relación parece menos evidente, pero las propiedades se podrían ver como el «lugar» (abstracto) en el que se localiza el sujeto: en Juan está enfermo / es inteligente, por ejemplo, se situaría a Juan en el ámbito de la enfermedad o de la inteligencia (cf. Juan está en coma / está comatoso). En algunas lenguas, como el galés o el dialonké, esta relación semántica tiene un reflejo muy claro en las similitudes formales, pues las cuatro estructuras se construyen con un SN sujeto y un SP en el predicado. Esto justifica que se hayan postulado análisis parecidos para todas ellas y, en muchos casos, que se analicen como variaciones superficiales de una misma estructura inicial.

Vistas las diferencias interlingüísticas por lo que respecta a las construcciones ALEP y dado que el catalán y el español son un caso relativamente marcado, se podría prever que los aprendices de catalán o de español tuvieran dificultades para construir este tipo de oraciones. El objetivo de este estudio es ver cómo influye la primera lengua en la adquisición de los verbos ser, estar, haber y tener del catalán y del español. No hemos querido limitarnos al tema clásico de la distinción entre ser y estar atributivos, sino que hemos pretendido ampliar el estudio a los otros tres tipos de construcciones, porque, visto el comportamiento de las lenguas implicadas y vistas las relaciones generales entre las cuatro construcciones, hemos creído que el problema iba mucho más allá de la atribución.

\subsection{Objetivos y delimitación del estudio}

Los objetivos de este estudio son, por una parte, presentar evidencias en favor de las hipótesis que sostienen que la primera lengua (L1) influye en la adquisición de las segundas (L2), pero que esto no implica que no existan estrategias generales de simplificación que, como han puesto de manifiesto muchos autores, actuarían de la misma manera en todos los aprendices, independientemente de la L1 y de la L2. Por otra parte, pretendemos mostrar que las relaciones entre las construcciones ALEP también se hacen evidentes en el proceso de adquisición de segundas lenguas y, finalmente, que algunos problemas que inicialmente se

\footnotetext{
${ }^{2}$ Usamos el término «cópula» para referirnos a todos estos tipos de verbos (cf. Pustet 2003 para más información sobre los tipos de elementos copulares en las diversas lenguas).
} 
podrían considerar específicos de un componente o de un aspecto de la gramática, en realidad están relacionados con otro.

El trabajo que presentamos se basa en un estudio transversal en el que se han analizado, fundamentalmente, las producciones erróneas ${ }^{3}$. No abordaremos el tema de los otros muchos factores externos que pueden afectar el proceso de adquisición de los hablantes. Usaremos el término «error» para referirnos a la producción que se desvía de la que es habitual en los nativos, dejando de lado las cuestiones normativas. No hemos incluido, pues, aquellos casos en los que, a pesar de desviarse de la norma establecida para cada L2, la producción es también usada por un porcentaje significativo de los nativos de la zona en la que el sujeto aprende la L2.

Las L1 de los hablantes estudiados son el árabe marroquí, el chino y el rumano. Se trata de lenguas que se comportan de modo distinto por lo que respecta a este tipo de construcciones, tanto entre sí como en relación con las L2.

\subsection{Muestra y procedimiento}

La muestra está constituida por la producción de 18 sujetos, tres aprendices adultos de catalán y tres de español para cada una de las L1, de entre 17 y 45 años, con un un nivel de L2 equivalente, en general, a un curso entre el básico 2 y el elemental 2, aunque hay alguno de nivel inferior en cada grupo. La mayoría de los sujetos habían recibido algún tipo de formación explícita para la adquisición de la L2 y todos residen en la provincia de Girona o en la comarca de Osona y están expuestos a datos de las dos L2. En su mayor parte, han sido localizados a través de las Escuelas de Adultos o del Consorci per a la Normalització Lingüística.

El procedimiento seguido ha sido la grabación, transcripción y codificación de entrevistas semiestructuradas con una parte narrativa/descriptiva elicitada a partir del material de Mayer 1969. La duración de las entrevistas es de entre 20 y 30 minutos. Para la transcripción y codificación se ha usado el sistema childes (MacWinney 2000).

Para los objetivos de este trabajo no hemos tenido en cuenta la diferencia entre los aprendices de catalán y los de español, puesto que para los temas tratados no se han observado, en general, grandes diferencias entre ellos. Sí hemos mencionado, sin embargo, aquellos aspectos en los que puntualmente parecía ser relevante la L2, como por ejemplo en las oraciones existenciales.

\footnotetext{
${ }^{3}$ Dado que no es un estudio longitudinal no podremos aportar más datos relativos a la evolución del proceso de adquisición que aquellos que se puedan extraer de las diferencias entre los hablantes de la muestra de niveles más avanzados y los más principiantes.
} 


\subsection{Antecedentes e hipótesis}

Partiendo del Análisis Contrastivo (Fries 1945, Weinreich 1953, Lado 1957), según el cual la lı ejerce una influencia negativa o interferencia especialmente cuando la L1 y la L2 son muy distintas, se podría predecir que los aprendices en cuya L1 no existe un verbo copulativo tendrían dificultades para incorporar uno de estos verbos en una L2 que los tuviera, como el español o el catalán, cosa que no debería suceder si la situación fuera la inversa. Sin embargo, estudios posteriores basados en el llamado Análisis de Errores (Corder 1967) demostraron que todos los aprendices de cualquier L2 tienden a elidir las cópulas, independientemente de cuál sea su L 1 y de si ésta tiene o no este tipo de elementos en su gramática. Así, los hablantes de español, como los de chino, también eliden la cópula to be cuando aprenden inglés (cf. Butterworth y Hatch 1978, Huang y Hatch 1978, Odlin 1989, etc.). Estos datos hicieron pensar en una estrategia de simplificación común para todos los aprendices y a negar que se deban a una influencia de la L1. Esto trajo consigo la propuesta de teorías que afirmaban que la transferencia de la L1 tiene un papel mínimo (si es que lo tiene) en la adquisición de una l2 y que son unos mecanismos cognitivos universales los que subyacen al aprendizaje de la L2; sería el sistema de la L2, y no el de la L1, el que guiaría este proceso (cf. Dulay, Burt y Krashen 1982, Krashen 1981, etc.).

Aparte de la elisión de be y la de have (como cópulas y como auxiliares) y de la posición de estos elementos en las secuencias de desarrollo en aprendices de inglés, los estudios sobre la adquisición de las estructuras atributivas, locativas, existenciales y posesivas no son muy numerosos. Entre los más recientes, hay que destacar los de van de Craats 2002 y van de Craats, van Hout y Corver 2002, sobre la adquisición de las oraciones posesivas del holandés por aprendices árabes y turcos. El tema más estudiado para el español es la distribución de los usos de sery estar en L2 (cf., por ejemplo, vanPatten 1987, Geeslin y Guijarro-Fuentes 2005, 2006) y en estudios más generales también se hace alguna referencia bastante aislada a los otros verbos (Vázquez 1991 y Fernández 1997). No conocemos estudios en los que se analicen conjuntamente los cuatro tipos de estructuras que hemos tomado en consideración en este trabajo.

La hipótesis básica de la que partimos es que los universales que se han propuesto en relación con la adquisición de las L2 pueden explicar algunas de las propiedades comunes de la producción de todos los aprendices independientemente de sus L1, pero que las diferencias tipológicas entre las lenguas implicadas (primeras y segundas lenguas) no pueden ser «transparentes» en el proceso de adquisición de la L2, puesto que en tal caso la producción correcta e incorrecta de todos los grupos sería muy similar (cf. Gass 1996, Odlin 2003, van de Craats 2002, van de Craats, van Hout y Corver 2002, etc.). Por tanto, partimos de la idea de que alguna parte de la gramática de la L1, en algún momento 
del proceso de adquisición, se transfiere a la L2; más concretamente, presumimos que, si bien en las primeras fases del proceso puede haber algunas similitudes entre las producciones de todos los aprendices (la elisión de las cópulas, por ejemplo), en fases posteriores las diferencias pueden ser mayores y los tipos de errores producidos deberían reflejar las diferencias gramaticales entre las L1 y las L2. Los límites a menudo imprecisos entre los cuatro tipos de construcciones analizadas también deberán reflejarse en la producción de los hablantes, que, atendiendo al funcionamiento de su L1, deberán tender a seleccionar un verbo «modelo» para cada una de ellas y a extenderlo a otras construcciones más marcadas en la L2.

\subsection{Estructura del estudio}

En el apartado 2 presentaremos el tipo de construcciones del catalán y del español en las que hemos centrado el estudio y en el 3, las características generales de los cuatro tipos de construcciones analizadas en cada una de las L1. En el cuarto apartado presentamos el análisis de la producción de los hablantes, con especial atención a las dificultades para construir oraciones ALEP y la relación entre las dificultades y las diferencias entre las L1 y las L2 y entre las distintas L1. Finalmente, en el quinto apartado exponemos las conclusiones sobre los datos analizados y sobre el papel de la L1 y de las estrategias universales de simplificación en la adquisición de una $\mathrm{L} 2$.

\section{LAS CONSTRUCCIONES ALEP EN CATALÁN Y EN ESPAÑOL: DELIMITACIÓN DE LOS CASOS ANALIZADOS}

Las construcciones ALEP pueden presentar distintas formas tanto en catalán como en español y, en muchos casos, los límites entre ellas distan mucho de ser claros y han sido motivo de debate en los estudios gramaticales de ambas lenguas. Hemos limitado los casos analizados a aquellos más representativos de cada uno de los tipos y que se dan con mayor frecuencia en el discurso de los aprendices de catalán y de español que constituye la muestra.

\subsection{Atributivas}

Hemos tomado en consideración las oraciones construidas con los verbos ser/estar con atributo adjetivo o participio, nombre sin determinante, sP, adverbio de modo o pronombre posesivo (Fernández Lebo- 
rans 1999). Se trata de oraciones caracterizadoras o clasificadoras ${ }^{4}$ :

(1) a. Ros4 Nosotros somos ortodoxos / ROT1 La majoria som ortodoxos b. ARs4 Yo soy comerciante / ART 1 Ells eren estudiants

c. ARs2 Yo soy de Marruecos / ROT3 Jo sóc d'Olot

d. ARs4 Esto es así / ART1 Això és així

e. Ros4 El niño está enfadado / XIT2 El nen està enfadat

f. - Juan está en coma / ROT3 Ara estic en estat

g. XIS3 (Esto) no está bien / ART2 Jo estic molt bé

h. - El libro es suyo / ART2 El sexe és teu

\subsection{Locativas}

El grupo de las locativas incluye las oraciones construidas con el verbo ser / estar en las que se afirma la localización de un sujeto determinado en un sitio o su permanencia en él; también hemos incluido en este grupo las oraciones con valor temporal, que suelen tener un sujeto que denota un evento, como en (2c) (cf. Fernández Leborans 1999, Ramos 2002):

(2) a. Ros5 La rana está en el bol / ART1 Quan jo era al Marroc

b. Ros3 Está aquí desde hace dos años / ROT3 Sempre he estat aquí

c. XIs 1 El Año Nuevo es más tarde que aquí / ART3 [el que passa a la pel-lícula] és durant la neteja ètnica

\subsection{Existenciales}

Hemos incluido en el grupo de las existenciales las oraciones con el verbo haber / haver-hi en las que se afirma la existencia de una entidad y, a menudo, se la sitúa en un determinado lugar. La entidad, objeto directo según la normativa y muchas veces sujeto en la lengua hablada, en español suele ser, aunque no siempre, un sN indefinido o un nombre escueto (cf. Leonetti 1999, Fernández y Táboas 1999); en catalán, en cambio, puede ser definido en muchos más contextos:

(3) a. XIs 1 En China hay una historia... / ART1 Allà hi ha molta gent b. Ros5 Aquí abajo hay agua / ART1 (Allà) no hi ha feina

c. - Habían dos coches / XiT 1 Hi han baralles

d. Ros 4 *Es que hay los reyes de Troya / ART3 Hi ha la Dolors Bramon

Tanto en español como en catalán, hay dos verbos más que pueden aparecer en contextos existenciales: el verbo estar en español cuando el SN es definido, como en (4), y ser / ser-hi en catalán cuando el locativo es inespecífico (Ramos 2002, p. 2000) o en alternancia con la forma haver$h i$, cuando no aparece ningún sN explícito, como en (5-6). Las dudas de

\footnotetext{
${ }^{4}$ Los sujetos de la muestra se identifican con tres letras y un número. El número corresponde al que se le ha asignado dentro del corpus; las dos primeras letras identifican a la L1 $(\mathrm{RO}=$ rumano, $\mathrm{AR}=$ árabe, $\mathrm{xi}=\mathrm{chino})$; la última letra señala l.a L2 $(\mathrm{s}=$ español, $\mathrm{T}=$ catalán).
} 
los hablantes nativos a la hora de valorar la gramaticalidad de (7) pone de manifiesto que los límites entre los usos de los verbos en su valor existencial y en el locativo son muy difíciles de trazar:

(4) a. Ros 5 Aquí adentro está la rata.

b. Ros3 [¿¿ué asignaturas te gustaban menos?]

- Sí, bueno, estaba la física...

(5) a. Aquests famosos documents, suposant que hi siguin, ningú no els ha vist mai.

b. - Que hi ha la teva mare? - Que hi ha algú?

- No, no hi és / *no hi ha - No, no hi ha ningú / *no hi és ningú

(6) XIT3 Tennis és una mica gran però a Xina no jugar aquest. És una pilota de goma.

(7) ARS2 (*/?) Después del Ramadán hay la fiesta del cordero

\subsection{Posesivas}

En el grupo de las posesivas hemos tomado en consideración las oraciones con el verbo tener / tenir en las que se expresa una relación de «pertenencia» (alienable o inalienable) entre dos entidades físicas o entre una entidad física y una abstracta:

(8) a. Ros5 Nosotros no tenemos coche / ART3 Aquest nen té una granota b. ARs3 Tiene miedo de las abejas / XIT2 El nen té molts poders

También hemos incluido en este grupo las estructuras con una doble predicación (oración reducida), tanto si el segundo predicado es adjetival (a veces con valor resultativo), como si es preposicional con valor locativo:

(9) a. - Él tiene los ojos hinchados / ROT2 Els tinc quasi tots gravats b. Ros4 Tengo una medalla de plata en casa / ART1 Tinc la dona aquí

\section{DeSCRIPCión DE LAS CONSTRUCCIONES EN LAS L1}

\section{1. Árabe marroquí}

En árabe marroquí, en tiempo presente, algunas de estas construcciones no presentan ningún verbo explícito. Como se puede apreciar en los ejemplos de (10), las atributivas se forman por yuxtaposición del sujeto y el atributo:

\footnotetext{
(10) a. had-əl-kəlb wāeər $\quad$ ATRIB.-1 (Caubet 1993, p. 41) este-el-perro malo Este perro es malo
} 


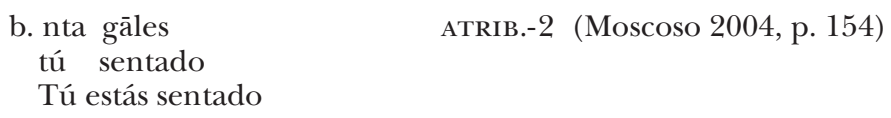

Por lo que respecta a las locativas, también puede haber yuxtaposición del sujeto (definido) y el locativo:

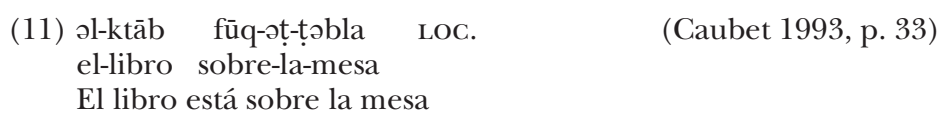

En algunas oraciones locativas con valor de un presente actual o en construcciones gnómicas, como las de $(12 \mathrm{a}, \mathrm{b})$, puede aparecer la forma verbal kāyn (participio activo de la cópula kāna), que también aparece en las existenciales:

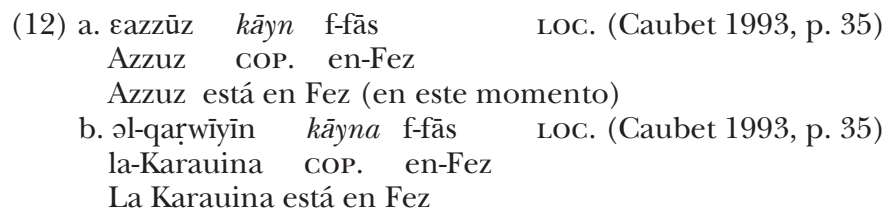

Las existenciales están estrechamente relacionadas con las locativas, pero el SN tiene valor indefinido y contienen siempre el participio activo de la cópula kāna:

(13) kāyn wāḥed-əl-ktāb fūq-əț-țabla $\quad$ ExIst. (Caubet 1993, p. 34) cop. un-el-libro sobre-la-mesa Hay un libro sobre la mesa

Cuando una oración con valor existencial contiene un SN con valor definido o que denota una entidad única, la construcción también incluye la cópula y el orden habitual es LOC - V - SN. En estos casos, en español se usaría el verbo estar y en catalán, haver-hi:

(14) f eš-šamāl kāyen el-bh $^{\circ} \mathrm{er}^{\circ}$ w f el-janūb kāyen eș-șehṛa en el-norte COP. el-mar y en el-sur COP. el-desierto $\mathrm{Al}$ norte está el mar y al sur está el desierto $\mathrm{Al}$ nord hi ha el mar i al sud hi ha el desert

(Quitout 1999, p. 98)

En las posesivas, el SN que denota la cosa poseída aparece precedido por el poseedor, que adopta la forma de un sintagma preposicional, con la preposición $\varepsilon a n d$ 'en, junto a' y un clítico pronominal sufijado. Para algunos autores, como Caubet 1993, este sintagma locativo está en fase muy avanzada de verbalización:

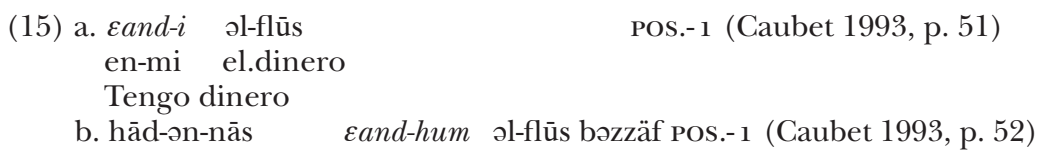


estas-las-personas en-ellas el-dinero.mucho

Estas personas tienen mucho dinero

La «posesión» de una sensación también se puede formular con una oración semejante a las anteriores pero con otra preposición:

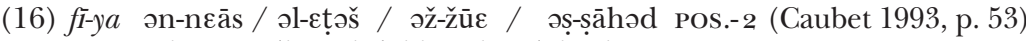
en-mi el-sueño/la-sed /el-hambre/el-calor

Tengo sueño / sed / hambre / calor

Cuando las oraciones no tienen valor de presente, todas contienen habitualmente el verbo kāna. La cópula concuerda con el sujeto de la atributiva y con el SN cuya locación, existencia o posesión se afirma (no con el poseedor, en las posesivas).

\subsection{Chino}

Las atributivas con adjetivo graduable (o verbo de estado) no suelen llevar ninguna cópula, como en (17a, b). Aparece la forma shi cuando se relaciona un sujeto referencial con un SN, como en (17c). En (17b) también se aprecia que cuando el predicado indica un cambio de estado aparece la partícula $l e$, la misma que se usa para el aspecto perfectivo:

(17) a. Tā (hěn) kāi xīn ATRIB.-1 (Li y Thompson 1987, p. 99) él (muy) feliz Él es feliz

b. Tā lèi le ATRIB.-2 él cansado PART. Él está cansado c. Wǒ shi xué shēng yo ser estudiante ATRIB.-3 (Lyovin 1997, p. 137) Yo soy estudiante

Las locativas se construyen con el coverbo zài. El SN aparece al inicio de la oración y se interpreta como definido:

(18) Qián zài zhèr $\quad$ LOC. (Ramsey 1987, p. 80)
dinero cov. aquí
El dinero está aquí

Las existenciales y las posesivas comparten la forma yǒu. En las existenciales el SN aparece después de yǒ u y se interpreta como indefinido (como en español). En las posesivas, el poseedor ocupa la posición inicial y la cosa poseída se sitúa después de yǒu. Nótese que en ambas el sintagma locativo (lugar o poseedor) aparece al inicio de la oración y el SN cuya existencia o posesión se afirma, en posición final; la «posesión» de una sensación se expresa con una estructura atributiva, como se ve en (20): 


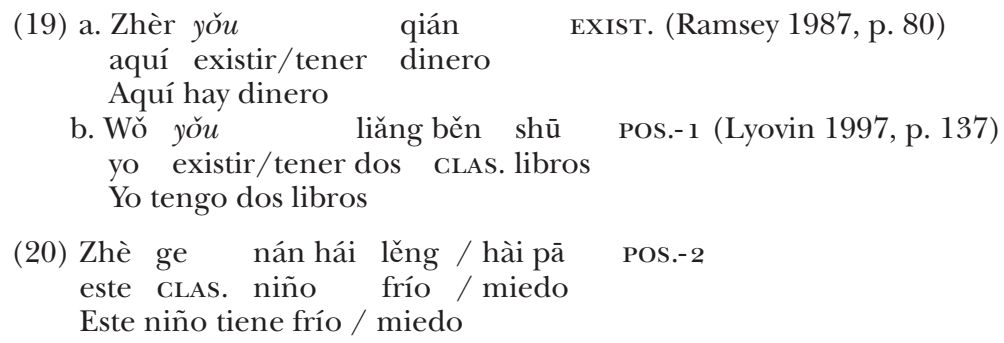

\subsection{Rumano}

En rumano un mismo verbo, $a f$, se usa en las atributivas, las locativas, las existenciales y una parte de las posesivas. En (21) presentamos ejemplos de oraciones atributivas (la forma de tercera persona singular del presente puede ser $e$ o este):
(21) a. Aceasta nu este recomandabil ATRIB.-1 (Avram 1997, p. 176) esto no es recomendable Esto no es recomendable
b. Prietenul $e$ bolnav ATRIB.-2 amigo-el está enfermo El amigo está enfermo

En las locativas el sujeto es definido y preverbal y en las existenciales el sujeto es posverbal e indefinido:
(22) a. Cartea e pe masă LOc. (Lombard 1974, p. 354)
libro-el es sobre mesa
El libro está encima de la mesa
b. $E$ pâine pe masă? $\quad$ EXIST. (Levițchi 1965, p. 228) es pan sobre mesa ¿Hay pan en la mesa?

Para expresar la posesión propiamente dicha existe el verbo a avea, como en (23a); para las que expresan la "posesión» de una sensación se utiliza el verbo a ficon el experimentante en dativo, como en (23b):
(23) a. $A i \quad$ permis de rezidență? Pos.-1 tienes permiso de residencia ¿Tienes permiso de residencia?
b. Nu-ți fie frică no+te sea miedo No tengas miedo
POS.-2 (Levițchi 1965, p. 228)

Una vez hecha esta sumaria presentación del comportamiento de las L1 tomadas en consideración, expondremos los resultados del análisis de los datos recogidos. 


\section{ANÁlisis DE LOS ERRORES PRODUCIDOS EN LAS CONSTRUCGIONES ALEP}

Los datos de referencia para este estudio son los recogidos en la tabla 1:

Tabla 1: Errores analizados por grupos y tipos de construcciones

\begin{tabular}{|c|c|c|c|c|}
\hline & ÁRABE & CHINO & RUMANO & TOTAL \\
\hline PROD.ANALIZADA & 401 & 333 & 452 & 1186 \\
\hline PROD. CORRECTA ${ }^{a}$ & $367(91,52)$ & $249(74,77)$ & $399(88,27)$ & $1015(85,58)$ \\
\hline TOTAL ERRORES ${ }^{\mathrm{a}}$ & $34(8,48)$ & $84(25,23)$ & $53(11,73)$ & $171(14,42)$ \\
\hline confusión $^{\mathrm{b}}$ & $10(29,41)$ & $20(23,81)$ & $36(67,93)$ & $66(38,60)$ \\
\hline omisión $^{\mathrm{b}}$ & $24(70,59)$ & $64(76,19)$ & $17(32,07)$ & $105(61,40)$ \\
\hline prod. total ${ }^{\mathrm{a}}$ & $154(38,40)$ & $169(50,75)$ & $216(47,79)$ & $539(45,45)$ \\
\hline errores $^{c}$ & $14(9,10)$ & $36(21,30)$ & $26(12,04)$ & $76(14,10)$ \\
\hline confusión $^{\mathrm{d}}$ & $2(14,29)$ & $4(11,11)$ & $16(61,54)$ & $22(28,95)$ \\
\hline omisión $^{\mathrm{d}}$ & $12(85,71)$ & $32(88,89)$ & $10(38,46)$ & $54(71,05)$ \\
\hline prod. total $^{\mathrm{a}}$ & $41(10,23)$ & $30(9,01)$ & $48(10,62)$ & $119(10,03)$ \\
\hline errores $^{c}$ & $3(7,32)$ & $9(30,00)$ & $9(18,75)$ & $21(17,65)$ \\
\hline confusión $^{\mathrm{d}}$ & $2(66,67)$ & $5(55,56)$ & $7(77,78)$ & $14(66,67)$ \\
\hline omisión $^{\mathrm{d}}$ & $1(33,33)$ & $4(44,44)$ & $2(22,22)$ & $7(33,33)$ \\
\hline prod. total ${ }^{\mathrm{a}}$ & $92(22,94)$ & $50(15,02)$ & $76(16,82)$ & $218(18,38)$ \\
\hline errores $^{c}$ & $11(11,96)$ & $25(50,00)$ & $13(17,11)$ & $49(22,48)$ \\
\hline confusión $^{\mathrm{d}}$ & $5(45,45)$ & $9(36,00)$ & $11(84,62)$ & $25(51,02)$ \\
\hline omisión $^{\mathrm{d}}$ & $6(54,55)$ & $16(64,00)$ & $2(15,38)$ & $24(48,98)$ \\
\hline prod. total ${ }^{\mathrm{a}}$ & $111(27,68)$ & $80(24,02)$ & $108(23,89)$ & $299(25,21)$ \\
\hline errores $^{c}$ & $3(2,70)$ & $10(12,50)$ & $1(0,93)$ & $14(4,68)$ \\
\hline confusión $^{\mathrm{d}}$ & $1(33,33)$ & $0(0,00)$ & $0(0,00)$ & $1(7,14)$ \\
\hline omisión $^{\mathrm{d}}$ & $2(66,67)$ & $10(100,00)$ & $1(100,00)$ & $13(92,86)$ \\
\hline OTROS ERRORES $^{5}$ & 3 & 4 & 4 & 11 \\
\hline confusión & 0 & 2 & 2 & 4 \\
\hline omisión & 3 & 2 & 2 & 7 \\
\hline
\end{tabular}

${ }^{\text {a }}$ Porcentaje sobre la producción total analizada del grupo. ${ }^{\mathrm{b}}$ Porcentaje sobre el total de errores del grupo. ${ }^{c}$ Porcentaje sobre la producción total analizada de cada tipo del grupo. ${ }^{\mathrm{d}}$ Porcentaje sobre el total de errores de cada tipo del grupo.

\footnotetext{
${ }^{5}$ Estos otros errores son casos en los que eran posibles dos interpretaciones y, por tanto, no se podian asignar a ningún grupo.
} 
En la tabla 1 se observa que el porcentaje de errores en estas construcciones es relativamente elevado ${ }^{6}$ y que en el grupo árabe y en el chino los errores de omisión son considerablemente más numerosos que los de confusión, a diferencia de lo que sucede en el rumano. También permite constatar que los hablantes de chino son los que producen un porcentaje mayor de errores en todas las construcciones; les siguen los que tienen el rumano como L1, que, excepto en las posesivas, han presentado un porcentaje de errores mayor que el de los árabes. En la misma tabla se pone de manifiesto que las construcciones que conllevan un mayor grado de dificultad son las existenciales, que para los árabes y los chinos suponen la mayor dificultad y para los rumanos, la segunda. Las posesivas, en cambio, son las que conllevan menos problemas para todos los grupos. Las dificultades de las locativas también son notables para el grupo chino y para el rumano, mientras que las atributivas proporcionalmente suponen, en general, menos problemas que las existenciales y las locativas. Como veremos más adelante, buena parte de los datos acabados de exponer se pueden explicar atendiendo a las características de la L1 de los hablantes.

A continuación presentaremos las características generales de la producción errónea de cada uno de los grupos de la muestra y su relación con las respectivas $\mathrm{L} 1$.

\subsubsection{El grupo árabe}

Como se observaba en la tabla 1, el grupo árabe ha producido 34 errores, 24 de los cuales eran de omisión (70,59\%), frente a sólo 10 de confusión $(29,41 \%)$. Excepto en un caso, todas las formas omitidas correspondían a un presente. La mayoría de los errores de confusión (tabla 2) se encuentran en las construcciones locativas y, especialmente, en las existenciales, que son las que les suponen más problemas. En la tabla 3, en cambio, se ve que el mayor número de errores de omisión se dan en oraciones atributivas, especialmente en las que deberían llevar el verbo ser.

Tabla 2: Errores de confusión (árabe) [10]

\begin{tabular}{|l|l|l|l|l|l|}
\hline ATRIB. & ser=estar & 1 & EXIS. & ser=haver-hi & 2 \\
\hline ATRIB & estar=ser & 1 & EXIS. & haber=estar & 3 \\
\hline LOC. & tenir=ser $/$ estar-se & 2 & POs. & haver-hi=tenir & 1 \\
\hline
\end{tabular}

Tabla 3: Errores de omisión (árabe) [24]

\begin{tabular}{|l|l|l|l|l|l|}
\hline ATRIB. & $0=$ ser & 10 & EXIS./POS. & $0=$ haber/tener & 2 \\
\hline
\end{tabular}

\footnotetext{
${ }^{6}$ Es más elevado, por ejemplo, que el que se ha encontrado en su flexión verbal, pero en los tres grupos se mantiene una correlación: los hablantes de árabe son los que producen menos errores $(5,13 \%$ de flexión y $8,48 \%$ en construcciones ALEP), seguidos de los rumanos $(7,76 \%$ y $11,73 \%)$ y de los hablantes de chino, los que producen más $(16,73 \%$ y $25,23 \%)$.
} 


\begin{tabular}{|l|l|l|l|l|l|}
\hline ATRIB. & $0=$ estar & 2 & POS.PRED. & $0=$ tenir & 2 \\
\hline LOC. & $0=$ estar & 1 & POS.LOC./EXIS & $0=$ tener/haber & 1 \\
\hline EXIS. & $0=$ haber & 6 & & & \\
\hline
\end{tabular}

Las construcciones que resultan más problemáticas para el grupo árabe son las existenciales. Se dan 6 casos de elisiones del verbo, como la de (24), cosa que resulta, a priori, poco previsible dado que en su L1 estas oraciones contienen la cópula kāna. El hecho de que casi todos los casos hayan sido producidos por los aprendices con un nivel más bajo de L2 sugiere que, en este caso, el origen del problema no habría que buscarlo en la L1 sino que se debería a la estrategia general de simplificación (elisión de las cópulas) ${ }^{7}$ :

(24) *LAP: sobretot allà: a l'hospital o $+\ldots$

*ART2: hospital sí.

*ART2: de urgències, sempre gent que $+\ldots$

*ART2: tenen problemes.

Hemos detectado también 5 construcciones en las que se ha usado un verbo por otro. En dos ocasiones se ha sustituido haver-hi por ser en catalán, como en (25), y en 3 más, haber en español ha sido utilizado en lugar de estar, cuando el SN era definido, como en (26):

(25) * ART2: e: després d'això era una xxx.

* ART2: era un avió que passa xxx.

(26) *ARS2: sí, hay el mar cerca de mí +...

En el uso de ser por haver-hi en (25) podrían verse implicadas varias circunstancias. Por una parte, es bastante general la sobreextensión de la cópula menos marcada ser (paralelo a su kāna), que, además, en catalán es el verbo usado en las locativas, y en la L1 de los aprendices las locativas y las existenciales pueden compartir la misma forma verbal. Y por otra parte, ser, como ya hemos apuntado anteriormente, también puede tener un uso existencial.

La sustitución de haber por estar en (26) está claramente relacionada con el hecho de que en español una existencial con el SN definido se construye con estar. Los aprendices que ya han adquirido el verbo prototípico haber tienden a generalizar su uso a todos los tipos de existenciales del español. En los tres ejemplos encontrados de este tipo (si vols el mar, hay el mar; después del Ramadán hay la fiesta del cordero), el sN era claramente definido y se refería a una entidad única; corresponderían a casos como el de (14) en su L1, que se traducirían con estar en español y con haver-hi en catalán. También cabría pensar en una posible influencia del catalán, puesto que los hablantes están expuestos a las dos L2. Se ve, pues, que existe un

\footnotetext{
${ }^{7}$ En los ejemplos hemos simplificado al máximo la transcripción para facilitar la lectura y hemos omitido algunos fragmentos irrelevantes para el estudio.
} 
problema para escoger un verbo de entre los cuatro de la L2 cuando en la L1 se usa la misma cópula que en el no-presente de las otras construcciones, y que también influyen propiedades muy específicas de la L2.

Las construcciones que representan menos problemas, como en los otros grupos, son las posesivas. Este hecho se podría relacionar con el valor más léxico de tener y con la estructura preposición + clítico poseedor de la L1, que ya es sentida como un paradigma verbal equivalente al verbo románico tener. En (27) presentamos los casos de omisión:

(27) a.*ART1: però últimament, m'he estabilitzat una mica i bueno tele: con una parabòlica $\mathrm{i}$ anem a veure a les dos versions.

b. *ART1: i la idea d'immigrar no la tenia [//] la idea no assumida.

(27b) es una construcción más compleja que la de (27a). Se trata de una oración reducida con un predicado adjetival, parecida a (28), que contiene un predicado locativo preposicional (tiene una botella en la cabeza) y que también podría interpretarse como una existencial (hay una botella en su cabeza). Estos límites poco claros en la L2 podrían dificultar la adquisición de estructuras de este tipo:

(28) *ARs 3: no sé si ha caído de la ventana o: no sé.

*ARS3: una botella en su cabeza...+

También se ha encontrado un caso poco claro de uso de haber (=haver-hi) por tenir.

(29) *ART2: el berber hay quatres: idiomes de berber diferents de idiomes

En cuanto a las locativas, que en la $\mathrm{L} 1$ de los aprendices no suelen llevar verbo, destaca un caso de omisión de estar, en (30):

(30) *ARS 2: no noche, no hay mucho ambiente.

*ARS 2: la gente en la calle, y ya está.

La inmensa mayoría de los errores en las atributivas, 12 de 14, son de omisión del verbo. El hecho de que en la L1 las atributivas en presente no lleven verbo parece una explicación clara para estos errores; sin embargo hay que tener presente también que todos han sido producidos por los aprendices de nivel más bajo. Cabría pues, en este caso, preguntarse hasta qué punto ha influido la lı y hasta qué punto se está aplicando una estrategia de simplificación general para todos los aprendices. En (31) presentamos dos ejemplos de omisión del verbo en atributivas:

(31) a. *ART 1: xxx per problemes d'aquest o a l'altre xxx que tothom igual dins la diversitat, no.

b. *ART 2: acollir, sí, muy muy acollidors, muy simpática es m:, en general, pero $<$ no $>[/]$ no bien la política.

Sólo en dos ocasiones se han confundido los dos verbos ser / estar de las atributivas en las L2 y hay que reconocer que son casos discutibles: 
(32) a. *ARS2:no puedo creer que la gente del pasado están como salvajes. b. *ART1: bueno, aquests són atrassats, són: +...

\subsubsection{El grupo chino}

En la tabla 1 se ha visto que de los 84 errores analizados del grupo chino, 64 eran de omisión (76,19\% del total analizado de su grupo) y había sólo 20 de confusión $(23,81 \%$ ). Los verbos confundidos (tabla 4) son, por una parte, ser y estar (en las dos direcciones, pero sobre todo $s e r=e s t a r)$ y, por otra parte, la sobreextensión del verbo tener a las oraciones existenciales. Las elisiones más frecuentes (tabla 5) son las que afectan a las oraciones atributivas (especialmente ser), pero también son numerosas las elisiones del existencial haber/haver-hi y, en menor grado, del posesivo tener/tenir.

Tabla 4: Errores de confusión (chino) [20]

\begin{tabular}{|l|l|l|l|l|c|}
\hline ATRIB. & ser=estar & 1 & EXIS. & tener=haber & 9 \\
\hline ATRIB. & estar=ser & 3 & EXIs. $/$ fer & tenir=haver-hi/fer & 1 \\
\hline LOC. & ser=estar & 5 & hacer (tiempo) & tener=hacer & 1 \\
\hline
\end{tabular}

Tabla 5: Errores de omisión (chino) [64]

\begin{tabular}{|l|l|c|l|l|c|}
\hline ATRIB. & $0=$ ser & 23 & EXIS. & $0=$ haver-hi & 12 \\
\hline ATRIB. & $0=$ estar & 7 & EXIS. & $0=$ haver-hi/ser & 1 \\
\hline ATRIB. & $0=$ estar/anar & 2 & EXIS/fer & $0=$ haver-hi/fer & 1 \\
\hline ATRIB. & $0=$ ser $/$ fer & 1 & POS. & $0=$ tener/tenir & 8 \\
\hline LOC. & $0=$ estar & 4 & POS.PRED. & $0=$ tener & 1 \\
\hline EXIS. & $0=$ haber & 3 & POS.LOC. & $0=$ tener & 1 \\
\hline
\end{tabular}

Como para el grupo árabe, para el grupo chino las existenciales son las construcciones que suponen mayores dificultades: un $50 \%$ de las producidas son erróneas. Se han detectado 9 casos de confusiones, todas de sustitución de haber/haver-hi por tener/tenir, como las dos de (33)

(33) a. *XIT1: en l'any: setanta: tenia una guerra allà, al sudest asiàtic.

b. *MAO: qué pasa en la película?

*xIs3: es que tengo una chica de baile muy bien y luego tienes una empresas tiene una chico.

La confusión entre los verbos haber/haver-hi y tener/tenir se puede dar esporádicamente en todos los grupos. El hecho de que en el grupo chino

\footnotetext{
${ }^{8}$ En el ejemplo de (33b) hay un problema añadido de construcción de relativas; la secuencia equivale a hay una chica que baila muy bien y luego hay un chico que tiene una(s) empresa(s).
} 
esta confusión sea especialmente frecuente parece innegable que está relacionado con las propiedades de la L 1 . En chino las posesivas y las existenciales, a diferencia de las otras construcciones, comparten una única forma verbal, yǒu, y, por tanto, a los aprendices les resulta más difícil distinguir los dos verbos usados en las lenguas románicas ${ }^{9}$. La tendencia de los aprendices chinos a sobreextender tener a haber podría explicarse por el hecho de que haber es sentido como un elemento funcional de flexión, que ellos eliden frecuentemente en la formación de tiempos compuestos, y, en cambio, tener es sentido como un verbo con más contenido léxico que pueden equiparar más fácilmente a su forma explícita yǒ u y realizar lo que para Freeze 1992 seria el rasgo locativo de Flexión.

Los errores más frecuentes en las existenciales, sin embargo, son de omisión del verbo, que se ha dado en 16 ocasiones:

(34) a. *xiT2: m:: y cuando yo era en China e:: en China coche poc, no, no como España, coche mucho, porque e autobús + /.

b. *XIT 1: sí, havia explicat perquè a Lao n'hi molta comunitat xinesos.

c. *XIT2: en Xina $h i$ : sud i nord $+/$.

Los casos más interesantes, sin duda, son los ejemplificados en (34b, c): son un tipo de error exclusivo del grupo chino y sucede en 7 de los 12 casos de omisión de haver-hi. En estas construcciones se ha reducido el verbo catalán haver-hi al clítico $h i$. No hay, pues, una verdadera omisión del existencial, sino que sólo se elide haver, el elemento que aporta las marcas de tiempo y de persona, que también se elide en las formas compuestas de los verbos. Haver es percibido como un elemento de flexión que los aprendices con una L1 aislante como el chino tienden a elidir también en la flexión verbal de la L2. El análisis de Freeze 1992 permite explicar fácilmente esta elisión del verbo y el mantenimiento del clítico $h i$ (una proforma existencial, en su terminología), puesto que considera que ésta no es más que la realización de un rasgo locativo de Flexión. Los hablantes que eliden haver de la forma existencial y mantienen hi no realizarían los rasgos de tiempo y concordancia, como en la L1 y en la flexión de la L2, pero sí el locativo, que correspondería a su forma yǒu.

Por lo que respecta a las posesivas, que, en parte, también incluyen la forma yó $u$ en la L1, son el grupo de construcciones que presentan un porcentaje más bajo de errores, como en los otros dos grupos lingüísticos. Todos estos errores son de omisión del verbo románico, como los ejemplificados en (35-37):

(35) *MAO: y qué, trabajas, todos los días?

*XIS4: sí.

*XIS4: ahora ya contrato agencia, sí.

\footnotetext{
${ }^{9}$ Otros autores también han detectado la confusión entre las formas posesivas y las existenciales en hablantes de chino. Valentini 1992, p. 193, observa que los aprendices chinos usan la forma existencial italiana c'` 'haber' en lugar de avere 'tener' (io c'è amici «yo tengo amigos»; lui c'è un moglie «él tiene una mujer») y en otros estudios se ha observado que la forma posesiva es la que se sobreextiende a la existencial (Huebner 1983, p. 176).
} 
(36) a. *XIs4: ah, sí, tirado esto, e:sto mucho, éste miedo para árbol sube b. ${ }^{\text {BEC: }}<$ ella $>[/]$ ella és molt bona persona $+\ldots$ *XIT2: sí, (...) aunque ell té moltes, moltes poder i <ella no> [/] però ella no miedo él.

(37) a. *xis3: y luego buscado la habitación de todos y la perrito e $<l a>[/ /]$ lo en cabeza dentro de la botella.

b. *MAO: caen, sí, se caen en el río.

*XIs4: niña con perro todo mojado ropa

El hecho de que en la L1 de estos aprendices las posesivas suelan contener una forma verbal no permitiría explicar por qué se dan tantas omisiones del verbo. Un análisis más detallado de los datos, sin embargo, permite explicar esta aparente paradoja. Por una parte, se dan sólo 2 casos como el de (35), donde se ha elidido el verbo tener en una construcción con un objeto directo que se refiere a una entidad física y los dos ejemplos han sido producidos por los dos hablantes de nivel más bajo en L2. Por consiguiente, se trataría de la aplicación de una estrategia de simplificación. Por otra parte, en los ejemplos en (36) no se expresa una posesión propiamente dicha sino la «posesión» de una sensación, y esto sucede en 6 de los 10 errores de omisión. Hay que recordar que este tipo de construcciones en chino no incluirían ninguna forma verbal, como las atributivas con predicado adjetival, que también suelen sufrir la omisión del verbo en la L2 (cf. 20). Finalmente, en (37) se ha elidido el verbo tener en construcciones más complejas que incluyen una predicación secundaria, locativa en (37a) y atributiva o resultativa en (37b): tiene la cabeza dentro de la botella y tiene toda la ropa mojada. Por tanto, podemos concluir que la elisión de tener/tenir puramente posesivo se reduce a sólo dos casos, los de (35). La existencia de la partícula china yǒu en la L1 y el valor más léxico de tener evitan la elisión del verbo románico cuando se ha superado la fase inicial.

En cuanto a las atributivas, de nuevo la casi totalidad de los errores $(32 / 36)$ son de omisión y sólo en cuatro casos se han confundido ser y estar:

(38) a. *xis 1: oído, oído xxx es muy bien, escucha no muy bien.

b. *MAO: y qué película fuiste a ver?

*xis3: no sé cómo se llama, que son, que sale baile, xxx está un poco famoso $\mathrm{xxx}$ no sabe $+\ldots$

Se han encontrado 23 omisiones de ser, como en (39), y 7 de estar, como en (40):

(39) a. *XIs3: es que:, este ternera con, cómo se dice, en China lo ternera más bueno que aquí y aqui un poco duro

b. *XIT2: en la seva casa té moltes diners.

*XIT2: la nena, no, molt pobra.

(40) a. *MAO: qué familia tienes?

*XIsı: e: madre y padre y hermano, hermana, pero mi hermana ya casada.

b. *XIT3: mira al darrere de un arbre.

*xIT3: perquè el arbre trencat. 
En chino, las atributivas con un «adjetivo» graduable no contienen ninguna forma verbal, posición en la que en las L2 pueden aparecer dos formas distintas. Esto explicaría las omisiones de (39) y las confusiones de (38). En (40), la forma elidida estar tiene como atributo una forma relacionada con un participio con valor perfectivo, de cambio de estado o resultativo. El verbo románico se siente aquí como un auxiliar que marca persona y tiempo y, por tanto, se elide, como sucede en las formas progresivas del tipo está cantando. El valor aspectual perfectivo, el rasgo pertinente en la L1, ya queda recogido en el atributo.

Finalmente, en las locativas, en las cuales producen un $30 \%$ de errores, se dan 5 casos de confusión y 4 de omisión. En las confusiones, se usa siempre ser en lugar de estar en español, como en (41), cosa que se podría relacionar tanto con la tendencia general a sobreextender la cópula ser como con el uso de ser en catalán en este tipo de estructuras, dado que los aprendices están expuestos a las dos L2:

(41) a. *XIs 1: pimiento es arriba de: e tierra, pero \# la cosa es a en la: e: tierra pero no sé como se llama $+\ldots$

b. *XIT2: en China cuando yo era en China y también en la escuela, también enseñar, un escuela $+/$.

Las omisiones, ejemplificadas en (42), pueden estar motivadas por el hecho de que en la L1 el sujeto y el locativo se enlazan por medio del coverbo zài, que se usa también como partícula preposicional con valor locativo. La aparición en las secuencias románicas de una preposición con este mismo valor (en) seguramente influye en la elisión del verbo:

(42) a. *XIT2: cuando yo en casa [//] mirar la tele

b. *MAO: si has viajado, has visitado algún otro país.

*XIs 1: yo sólo en e: francés. [=Francia]

\subsubsection{El grupo rumano}

En la tabla 1 se ha constatado que de los 53 errores analizados del grupo rumano, sólo 17 eran de omisión (32,07\%), frente a 36 de confusión $(67,93 \%)$. Estos datos contrastan claramente con los del grupo árabe y los del grupo chino. Por lo que concierne a las confusiones, como se ve en la tabla 6 , el verbo que se sobreextiende más es estar (a las atributivas [14] y a las existenciales y locativas [2/3]). También es muy frecuente el uso erróneo de ser: en 5 casos por estar locativo, en 2 por estar atributivo y en 7 por haver-hi. Si bien en las atributivas las confusiones son sólo entre sery estar, las locativas incorrectas se han construido con ser o con haber y tener (temporal), y las existenciales con cualquiera de los cuatro verbos (nótese la diferencia con los verbos confundidos mayoritariamente en el grupo chino: haber / tener): 
Tabla 6: Errores de confusión (rumano) [36]

\begin{tabular}{|l|l|c|l|l|l|}
\hline ATRIB. & ser=estar & 2 & EXIS./LOC. & estar=ser & 1 \\
\hline ATRIB. & estar=ser & 14 & EXIS. & ser=haver-hi & 7 \\
\hline ATRIB./EXIS. & estar=ser/haber & 1 & EXIS. & tener=haver-hi & 2 \\
\hline LOC. & ser=estar & 5 & EXIS. & haber=estar & 1 \\
\hline LOC.T & haber=ser & 1 & EXIS. & estar=haber & 1 \\
\hline LOC.T & tener=ser & 1 & & & \\
\hline
\end{tabular}

Los casos de omisión son menos frecuentes y se concentran especialmente en la omisión de ser en las atributivas:

Tabla 7: Errores de omisión (rumano) [17]

\begin{tabular}{|l|l|c|l|l|l|}
\hline ATRIB. & $0=$ ser & 10 & EXIs. & $0=$ haber & 2 \\
\hline ATRIB. $/$ hacer & $0=$ ser/hacer & 1 & POS. & $0=$ tenir & 1 \\
\hline LOC. & $0=$ estar & 2 & POS./hacer & $0=$ tener/hacer & 1 \\
\hline
\end{tabular}

En el grupo rumano, las construcciones que resultan más problemáticas son las locativas y las existenciales, y en ambos casos la gran mayoría de los errores son de confusión. En el caso de las locativas no temporales, se sustituye 5 veces estar por ser:

(43) a. * Ros5: media año camarero y: el mes pasado ya hace un año de cuando soy en esa fábrica de carne, xxx con carne de cerdo.

b. $*_{\mathrm{Ros}}$ : el chico no es, ah, con las abejas mira, que ya ha salido muchas $+\ldots$

Las confusiones en las existenciales son más variadas. En 9 ocasiones se reemplaza haver-hi/haber por ser/estar, como en (44a, b), y una vez se usa haber en lugar de estar en español, como en (44c):

(44) a. *Rot3: (...) perquè abans de Setmana Santa són sis setmanes que a casa meu es fa com una quaresma aquí.

b. * Ros5: últimamente que domingo está más trabajo, te vas a la once, a las doce (...).

c. *MAO: hm, y la historia qué hay, cuántos protagonistas hay?

* MAO: es de amor, tiene que haber por lo menos dos $+\ldots$

*Ros4: sí, es que hay <los> [/] los reyes de Troya, el viejo y los dos hijos, que uno de los hijos se enamora de la mujer de un rey griego, y lo coge y se va para Troya y el marido va.

Si se tiene en cuenta que en rumano las locativas y las existenciales se construyen con un mismo verbo, $a$ fi, que es el que también aparece en las atributivas, el origen de los errores como los de (44a, b) parece bastante ligado a la $\mathrm{L} 1 \mathrm{de}$ los aprendices. El caso de (44c) se explica porque cuando el SN es definido la L2 utiliza el verbo estar en las existenciales en lugar del prototípico haber, que es el que el aprendiz ha sobregene- 
ralizado. Dos factores más podrían haber propiciado esta confusión: la exposición de los aprendices al catalán, que usa el verbo haver-hi en estos contextos, y el uso de haber en la pregunta del entrevistador, que puede haber sido recuperado por el aprendiz.

En cuanto a las omisiones en las locativas y las existenciales, ya se ha dicho que eran mucho menos numerosas que las confusiones, lo que se explica porque en la L1 estas frases siempre contienen un verbo. Sólo se han detectado 4 casos de elisión de estar o de haber como los de (45), que han sido producidos por el aprendiz con un nivel más bajo de L2, cosa que hace pensar que estaba recurriendo a una estrategia de simplificación elidiendo la forma verbal:

(45) a. * Ros5: y: primera vez me encontrá camarero, fui media año camarero y después en este fábrica, pero antes de camarero dos semanas en una fábrica de xxx en xxx.

b. *Ros5: también juega, música muy buena a mí, te diré una cosa, la música de mi país está muy buena,, eh.

Por lo que respecta a las atributivas, también las confusiones son más numerosas que las omisiones. Hay 10 casos de elisión del verbo (siempre ser) frente a 16 casos de confusión:

(46) a. *MAO: y cuál es tu trabajo?

*Ros5: carnicero, mira, va a trabajar también con jamones, sabes, y también con, no sé, esto muy difícil, cabezas de cerdo.

b. *ROS5: mi cuñada hace muchas comidas de su país.

*ROS5: y casi com Rumanía sabes,, pero ahora vivo solo (...)

Este tipo de errores son menos frecuentes que las confusiones porque la L1 contiene siempre una forma verbal, cosa que en estadios no iniciales evita la elisión. Todas las omisiones han sido producidas por el hablante que tenía el nivel más bajo de L2, lo que lleva a pensar en la aplicación de una estrategia de simplificación general.

Las confusiones se dan sobre todo en la utilización de estar por ser, en 14 ocasiones, y mucho menos, sólo 2 veces, en la sustitución de estar por ser. Ejemplificamos estos casos en (47) y (48), respectivamente:

(47) a. *Ros5: la música de mi país está muy buena,, eh.

b. *Ros 3 : m:, mi madre está húngaro y también en mi casa de Rumanía se habla en húngaro.

c. * Ros 5 : la jefa del bar donde fui yo camarero estaba de Perú.

(48) * Ros5: xxx, vale, mira, es que te digo, yo soy loco por la música $<$ de mi $>$ [/] de mi país que $+/$.

La sobreextensión de estar a ser (y no viceversa, que es lo más frecuente) puede estar relacionada con las formas de 2ps y 3 ps del presente en la L1 (eşti y éste), fácilmente asimilable al verbo estar. La mayor parte de los errores se dan en estas dos formas. 
Finalmente, sólo se ha encontrado un error en las posesivas, que es el caso de omisión de (49):

(49) *LAP: $<$ i amb els amics $>$ [//] tens amics aquí?

*ROT2: sí, els amics molt de vergonya de parlar català.

El rumano tiene un verbo para las posesivas, a avea, que es distinto del de las otras construcciones. Esto influye positivamente en la corrección de las posesivas producidas por el grupo rumano. La elisión del verbo en (49) hay que relacionarla con el hecho de que en la L1 estas oraciones que expresan la "posesión» de una sensación no se construyen con a avea sino con $a$ fi, que es el verbo que se elide con más frecuencia.

\section{Conclusiones}

Aunque la elisión de la cópula (ser/estary también haber) sea un error general en la adquisición de cualquier lengua, cosa que no se podría predecir directamente de un análisis contrastivo, los datos presentados en este estudio permiten concluir que el funcionamiento de las estructuras analizadas en la L1 de los hablantes sí influye en la adquisición de la L2. El hecho de tratar conjuntamente estas cuatro construcciones, que en otras lenguas están mucho más relacionadas de lo que aparentemente lo están en catalán y en español, ha permitido ver que es necesario recurrir a una explicación basada en la transferencia de la l 1 para explicar el comportamiento de los aprendices estudiados y las diferencias que se detectan entre los grupos en estadios no iniciales.

Aunque se ha visto que las características de las L2 y la relación entre las cuatro construcciones en todas las lenguas pueden influir en el proceso de adquisición ${ }^{10}$, creemos que hay otros dos factores que son mucho más relevantes. Nuestro objetivo era observar la influencia de la L1 y la de la estrategia general de simplificación en la adquisición de las construcciones ALEP del catalán y del español. A continuación expon-

\footnotetext{
${ }^{10}$ Muy sumariamente, mencionamos los casos más destacados: a) La existencia de dos verbos distintos (sery estar) en las L2 para las oraciones atributivas provoca algunos errores de confusión. b) El hecho de que en las L2 haya un único verbo para las posesivas (tener/tenir) reduce el número de errores de confusión debidos a la L2. c) El verbo de la L2 tener/tenir se percibe con un contenido léxico mayor que ser / estar / haber/haver-hi, que se sienten más relacionados a los elementos gramaticales de flexión verbal; dado que los elementos con contenido léxico se aprenden antes que los que tienen un valor meramente funcional (cf. Fernández 1997, van de Craats, van Hout y Corver 2002, etc.), la diferencia entre haber y tener podría explicar por qué se detectan más dificultades en las existenciales que en las posesivas a pesar de que en ambas construcciones las tres L1 tienen alguna forma verbal. d) Las dos L2 a las que están expuestos se comportan parcialmente de manera distinta, cosa que puede dificultar el proceso de adquisición de los verbos. Ye) las existenciales en español, a diferencia de lo que sucede en catalán, difícilmente admiten el verbo existencial prototípico haber cuando el SN es definido, en cuyo caso se usa estar, que también aparece en las locativas (que se construyen con ser en catalán); todo ello provoca errores de confusión.
} 
dremos las conclusiones a las que hemos llegado a partir del análisis de los datos recogidos en nuestra muestra, cuyas limitaciones (cantidad de datos, de lenguas implicadas y carácter transversal del estudio) hay que tener presente en todo momento.

Por lo que concierne a la estrategia general de simplificación, aunque existe alguna dificultad metodológica de la hipótesis universalista según la cual la elisión de las cópulas es un fenómeno general en la adquisición de lenguas (cuando en la L1 no hay verbo, ¿están aplicando esta estrategia o están recurriendo a su L1 ?), esta hipótesis no ha sido desmentida, puesto que los hablantes con un nivel más bajo de L2, independientemente de su L1, son los que producen el mayor número de elisiones incorrectas.

En cuanto a la L1, hay propiedades del discurso de los aprendices que sólo se pueden explicar partiendo de su influencia en el proceso de adquisición de la L2. Tanto las características de las construcciones ALEP como las de la flexión de la lı influyen en la adquisición de estas oraciones de la L2. A modo de resumen, mencionaremos los casos más representativos que hemos señalado a lo largo de la exposición que demuestran que hay diferencias entre las producciones de los tres grupos que se deben explicar partiendo de sus respectivas L 1 .

En primer lugar, la cantidad de errores de cada grupo ya es un dato significativo: el grupo chino es el que presenta un porcentaje más elevado en su producción, seguido del rumano y del árabe. Dado que es el árabe y no el rumano el grupo que produce menos errores, se deduce que lo que causa más dificultades no es el hecho de carecer de verbo en buena parte de las construcciones (formas nuevas, en la terminología de Stockwell, Bowen y Martin 1965). Se debería pensar que es más complicado distinguir formas distintas en la L2 para lo que en la L1 es una sola forma, es decir, los casos de desdoblamiento (el grupo rumano produce más errores que el árabe pero menos que el chino). En el caso del chino (el que comete más errores de los tres grupos) se dan ambas circunstancias, formas nuevas y desdoblamiento. La morfología aislante del chino, además, también influye en algunos casos de omisión, concretamente en las de estary haber, verbos que coinciden con los auxiliares de las formas verbales compuestas o perifrásticas (ha cantado / está cantando) y que tienen un contenido semántico muy débil. Estas formas se eliden del mismo modo que sucede en la flexión - ah, sí, tirado esto; ella mirando; (cf. Gràcia 2007) - en el caso de los aprendices chinos estas elisiones suponen un 15,44\% [19] de sus errores de flexión verbal, mientras que para los rumanos el porcentaje es sólo del 1,08\% [1].

En segundo lugar, también es relevante la diferencia entre el porcentaje de errores de elisión respecto a los de confusión entre el grupo rumano por una parte y el chino y el árabe por otra. La existencia de verbos en la L1 en todas las construcciones (rumano), además del tipo de 
morfología verbal, influye en el hecho de que en estadios intermedios la tendencia inicial a elidir estos elementos se reduzca considerablemente, es decir, que se supere antes la fase de elisión que en los casos en los que la $\mathrm{L} 1$ carece total o parcialmente de cópulas.

En tercer lugar, la confusión entre los verbos existenciales y los posesivos en el grupo chino sólo se puede explicar si se tiene en cuenta que el chino posee una única forma para las existenciales y para un tipo de posesivas. En estos casos pueden elidir haber o la parte verbal de haver-hi, como hacen en la flexión verbal, o bien pueden usar el verbo tener (no asimilable a una marca de flexión) tanto para las existenciales como para las posesivas. Este comportamiento del grupo chino contrasta con el de los hablantes árabes y rumanos, que tienen una L1 con una flexión verbal rica como la de las L2, y en las que no hay un sólo verbo (o elemento equivalente) para las existenciales y para las posesivas.

En cuarto lugar, se ha visto que las construcciones posesivas son las menos problemáticas para todos los grupos. Esto está relacionado con el hecho de que en las tres lenguas las posesivas prototípicas contienen algún elemento relativamente equiparable a un verbo, cosa que evita errores de elisión. Las elisiones del verbo tener están muy restringidas en todos los grupos a las oraciones que indican una sensación: en las L1 estas construcciones no tienen verbo o contienen la cópula que se elide con mayor frecuencia.

En quinto lugar, la sobreextensión de estar a ser en el grupo rumano se justificaría por la existencia en la conjugación de su cópula $a f i$ de raíces equivalentes a la de estar. La mayoría de estos errores se dan en la tercera y en la segunda persona del singular del presente de indicativo que son, precisamente, las que en rumano tienen la raíz est-.

Finalmente, si bien se ha demostrado que también existe una estrategia de simplificación inicial en la adquisición de la flexión (cf. Bybee 1991, Perdue 1991, Andersen 1991, Andersen y Shirai 1996, Klein y Perdue 1997, etc.), para explicar la relación entre la omisión de los verbos románicos ser, estary haber en estas construcciones y en la flexión verbal es necesario apelar a las propiedades de las L1. Las diferencias entre las lenguas implicadas son básicas para dar cuenta de las diferencias que se dan en estadios más avanzados del proceso, cf. Gràcia y Serrat 2003; Gràcia 2007; y Serrat, Gràcia y Perpiñà 2007. Los hablantes de una lengua aislante como el chino tienen más problemas que los otros dos grupos, con L1 flexivas, tanto en la flexión verbal como en el uso de los verbos con los que se construyen las oraciones analizadas en este trabajo. Uno de los ejemplos más claros de esta relación es la omisión por parte de los aprendices chinos de catalán de la parte verbal de haver-hi y el mantenimiento de la proforma existencial $h i$, que correspondería a la forma invariable yǒ u de la L1. Este error aparece únicamente en los datos del grupo chino. 
En el futuro, este estudio se debería ampliar con el análisis de un abanico de L1 más amplio que cubra más lenguas tipológicamente distintas en este aspecto y con otro estudio centrado en algunas variedades románicas (francés, italiano, portugués, etc.) para observar qué sucede cuando las lenguas son mucho más parecidas. Este trabajo, pues, es un primer paso hacia un estudio más extenso que permita explicar la relación entre las diferencias tipológicas y la adquisición de las construcciones ALEP en segundas lenguas.

\section{REFERENCIAS BIBLIOGRÁFRICAS}

Allen, W. S. (1964): «Transitivity and possession», Language 40, pp. 337-343.

Andersen, R. W. (1991): «Developmental sequences: the emergence of aspect marking in second language acquisition», en Huebner, T.y Ferguson, Ch. A. (eds.), Crosscurrents in second language acquisition and linguistic theories, Ámsterdam, John Benjamins, pp. 305-324.

— y SHirai, Y. (1996): «The primacy of aspect in first and second language acquisition: the pidging-creole connection", en Ritchie, W. C. y Bathia, T. K. (eds.), Handbook of second language acquisition, Nueva York, Academic Press, pp. $527-570$.

Avram, M. (1997): Gramatica pentru toți, 2ª ed., Bucarest, Humanitas.

BACH, E. (1967): "Have and be in English syntax», Language 43, pp. 462-485.

Benveniste, É. (1950): «La phrase nominale», Bulletin de la Société de Linguistique de Paris 46. [también en É. Benveniste 1966: Problèmes de Linguistique Générale I, París, Gallimard, pp. 151-167].

- (1960): «Etre et avoir dans leurs fonctions linguistiques», Bulletin de la Société de Linguistique 55. [también en É. Benveniste 1966: Problèmes de Linguistique Générale I, París, Gallimard, pp. 187-207].

ButTer worth, G. y HATCH, E. (1978): «A Spanish-speaking adolescent's acquisition of English», en Hatch, E. (ed.), Second language acquisition, a book of readings, Rowley, Mass., Newbury House.

Bybee, J. (1991): «Natural morpholgy: the organization of paradigms and language acquisition», en Huebner, T. y Ferguson, Ch. A. (eds.), Crosscurrents in second language acquisition and linguistic theories, Ámsterdam, John Benjamins, pp. 67-91.

Caubet, D. (1993): L'Arabe marocain. syntaxe et catégories grammaticales, Textes, vol. II, París, Éditions Peeters.

Clark, E. V. (1978): «Locationals: existential, locative and possessive constructions», en Greenberg, J. H. (ed.), Universals of human languages IV (Syntax), Standford, Standford University Press, pp. 85-126.

Corder, S. P. (1967): «The significance of learners errors», International review of applied linguistics 5, pp. 161-170.

CraATs, I. van de (2002): «Conservation in the acquisition of possessive lexical items», Linguistics in the Netherlands 2002, pp. 37-47.

— van Hout, R. y Corver, N. (2002): «The acquisition of possessive have-clauses by Turkish and Moroccan learners of Dutch», Bilingualism: language and cognition 5, pp. 147-174. 
Dulay, H., Burt, M. y Krashen, S. (1982): Language two, Nueva York, Oxford University Press.

FERNÁNDEZ, S. (1997): Interlengua y análisis de errores en el aprendizaje del español como lengua extranjera, Madrid, Edelsa.

FernÁndez, O. y TÁboAs, S. (1999): «Construcciones impersonales no reflejas», en Bosque, I. y Demonte, V. (dirs.), Gramática descriptiva de la lengua española, vol. 2, Madrid, Espasa, pp. 1723-1778.

Fernández Leborans, M. J. (1999): «La predicación: Las oraciones copulativas», en Bosque, I. y Demonte, V. (dirs.), Gramática descriptiva de la lengua española, vol. 2. Madrid, Espasa, pp. 2357-2460.

Fillmore, Ch. J. (1966): «Towards a modern theory of case». The Ohio State University project on linguistic analysis, Repport Number 13, pp. 1-24.

Freeze, R. (1992): «Existential and other locatives», Language 68, pp. 553-595.

FrIEs, Ch. (1945): Teaching and learning English as a foreign language. Ann Arbor, University of Michigan Press.

Gass, S. (1996): «Second language acquisition and linguistic theory: The role of language transfer» en Ritchie, A. W. y Bhatia, T. (eds.), Handbook of second language acquisition, Nueva York, Academic Press, pp. 317-345.

Geeslin, K. L. y. Guijarro-Fuentes, P. (2005): «The acquisition of copula choice in instructed Spanish: the role of individual characteristics», en Eddington, D. (ed.), Selected proceedings of the 6th conference on the acquisition of Spanish and Portuguese as first and second language, Somerville, Mass., Cascadilla Proceedings Project, pp. 66-77.

Geeslin, K. L. y Gujjarro-Fuentes, P. (2006): «Second language acquisition of variable structures in Spanish by Portuguese speakers», Language learning 56, pp. 53-107.

GRÀcia, L. (2007): «Diferencias tipológicas y adquisición de segundas lenguas: flexión verbal y orden sintáctico», Revista Española de Lingüistica 37 (en prensa).

- y Serrat, E. (2003): «Immigració i adquisició de segones llengües: una aproximació als errors en la morfologia verbal», Caplletra 35, pp. 153-168.

Huang, J. y Hatch, E. (1978): «A Chinese child's acquisition of English», en Hatch, E. (ed.), Second language acquisition. A book of readings, Rowley, Mass., Newbury House.

Huebner (1983): A longitudinal analysis of the acquisition of English. Ann Arbor, Karoma.

JACKENDOFF, R. (1974): «A deep structure projection rule», Linguistic Inquiry 5, pp. 481-505.

- (1976): «Toward an explanatory semantic representation», Linguistic Inquiry 7, pp. 89-150.

Klein, W. y Perdue, C. (1997): "The basic variety», Second language research 13, pp. 301-347.

Krashen, S. (1981): Second language acquisition and second language learning, Oxford, Pergamon.

LADO, R. (1957): Linguistics across cultures: applied linguistics for language teachers, Ann Arbor, University of Michigan.

Leonetti, M. (1999): «El artículo», en Bosque, I. y Demonte, V. (dirs.), Gramática descriptiva de la lengua española, vol 1, Madrid, Espasa, pp. 787-890. 
Levițchi, L. (1965): Dicționar român - englez, Bucarest, Editura Ştiințifică.

Li, Сh. y Thompson, S. A. 1987: «Chinese», en Comrie, B. (ed.), The major languages of East and South-East Asia, Londres, Routledge, pp. 83-105.

Lombard, A. (1974): La langue roumaine. Une présentation, París, Klincksieck.

Lyons, J. (1967): "A note on possessive, existential and locative sentences», Foundations of languages 3, pp. 397-402.

Lyovin, A. (1997): An introduction to the languages of the world, Oxford, Oxford University Press.

MacWinney, B. (2000): The Childes project, Hillsdale, N. J., Erlbaum.

Mayer, M. (1969): Frog, where are you?, Nueva York, Dial Books for Young Readers.

Moscoso, F. (2004): Esbozo gramatical del árabe marroquí, Cuenca, Universidad de Castilla-La Mancha.

Oduin, T. (1989): Language transfer, Cambridge, Cambridge University Press.

Oblin, T. (2003): «Cross-linguistic influence», en Dougthy, C. J. y Long, M. H. (eds.), The handbook of second language acquisition, Oxford, Blackwell, pp. 436-481.

Perdue, C. (1991): «Cross-linguistic comparisons: organizational principles in learner languages», en Huebner, T. y Ferguson, Ch. A. (eds.), Crosscurrents in second language acquisition and linguistic theories, Amsterdam, John Benjamins, pp. 405-422.

Pustet, R. (2003): Copulas. Universals in the categorization of the lexicon, Oxford, Oxford University Press.

Quitout, M. (1999): Initiation à l'arabe maghrébien, París, L'Harmattan.

Ramos, J. R. (2002): «La predicació verbal no obligatòria», en Solà, J. (dir.), Gramàtica del català contemporani, vol. 2, Barcelona, Empúries, pp. 1951-2044.

Ramsey, S. R. (1987): The languages of China, Princeton, N. J., Princeton University Press.

Serrat, E., Gràcia, L. y Perpiñà, L. (2007): «First languages influence in second language acquisition: the case of immigrant L1 Soninke, Tagalog and Chinese children learning Catalan», en Pérez-Vidal, C. y otros (eds.), A portrait of the young in the new multilingual Spain, Multilingual Matters, Clevedon.

Stockwell, R., Bowen, J. D. y Martin, J. (1965): The grammatical structures of English and Spanish, Chicago, University of Chicago Press.

Valentini, A. (1992): L'italiano dei cinesi, Milán, Guerini.

VanPatten, B. (1987): «The acquisition of ser and estar. Accounting for developmental patterns», en VanPatten, B., Dvorak, T. y Lee, J. (eds.), Foreign language learning: a research perspective, Nueva York, Newbury House, pp. 61-75.

VÁzQuez, G. E. (1991): Análisis de errores y aprendizaje de español/lengua extranjera, Frankfurt, Peter Lang.

WeInReich, U. (1953): Languages in contact, La Haya, Mouton. 
